\title{
The anisotropic properties of a terbium-based liquid crystal complex
}

\author{
L. A. Dobrun ${ }^{1}$, A. S. Sakhatskii ${ }^{1}$, A.P. Kovshik ${ }^{1}$, E. I. Ryumtsev ${ }^{1}$, W. Haase ${ }^{2}$, \\ Yu. G. Galyametdinov ${ }^{3}$, A. S. Krupin ${ }^{3}$, A. A. Knyazev ${ }^{3}$ \\ ${ }^{1}$ Saint Petersburg State University, Universitetskaya nab. 7/9, Saint Petersburg, 199034, Russia \\ ${ }^{2}$ Technical University Darmstadt, Alarich-Weiss-Str 4, Darmstadt, D 64284, Germany \\ ${ }^{3}$ Kazan National Research Technological University, Karl Marks street 68, Kazan, 420015, Russia \\ 1.dobrun@spbu.ru
}

DOI 10.17586/2220-8054-2016-7-6-911-918

\begin{abstract}
The dielectric properties of a paramagnetic terbium-containing liquid crystal have been studied. The magnitude and the sign of the dielectric of the liquid crystalline complex have been determined. The relaxation processes (modes) characterizing the dispersion of the principal values of the sample's dielectric permittivity has been studied. The relaxation times, activation energy and the dipole moment of the complex could be evaluated.
\end{abstract}

Keywords: lanthanidomesogens, dielectric permittivity, dielectric anisotropy.

Received: 14 October 2016

Revised: 20 November 2016

\section{Introduction}

Lanthanide containing compounds are widely used in various optical electronic devices, organic light-emitting diodes of different colors, flat-panel and flexible displays, optical waveguides, hybrid lasers, solar cells, etc [1-3]. Synthesizing and studying the molecular and macroscopic properties of liquid crystalline coordination compounds of lanthanides (lanthanidomesogens) is an important physicochemical problem. Paramagnetic complexes of lanthanides with organic ligands combined highly effective luminescence with abnormally large values of magnetic susceptibility anisotropy, allowing the easy alignment under the influence of an external magnetic field [4]. The technical characteristics of the lanthanidomesogens depend essentially on their physical properties.

Some important physical parameters that determine the effectiveness of the orientation of the lanthanidomesogens under the magnetic or electric field are the anisotropy of magnetic susceptibility and of dielectric anisotropy, respectively. Physical characteristics of lanthanide coordination compounds are determined by the central ion and its surrounding ligands [5]. Therefore, studying the relationship between molecular and macroscopic properties of lanthanidomesogens is an important task of physical chemistry of the lanthanides.

In recent years, a number of lanthanide complexes that include Lewis bases and -diketones serving as ligands have been synthesized [6-10]. Rare examples of these compounds have a stable enantiotropic nematic phase within a wide temperature range, which was demonstrated by methods of differential scanning calorimetry and polarizing optical microscopy $[11,12]$. It was found that magnetic properties of lanthanidomesogens are strongly dependent upon the nature of the lanthanide ion and on the structure of the coordination center [13,14]. The first study of the dielectric properties of lanthanidomesogens - tris[1- (4- (4-propylcyclohexyl) phenyl) octane-1,3-dion][5,5'-diheptadecyl-2,2'-bipyridine] ytterbium $\left(\mathrm{Yb}\left(\mathrm{CPDk}_{3-5}\right)_{3} \mathrm{Bpy}_{17-17}\right)$ is presented in [15, 16]. The frequency dependence of the components of the dielectric permittivity tensor has been obtained in the range between $100 \mathrm{~Hz}-$ $5 \mathrm{MHz}$. The magnitude and the sign of the dielectric anisotropy, the relaxation time, the activation energy, and the dipole moment have been determined.

The aim of this study is to investigate the influence of the central ion on dielectric properties of nematic lanthanidomesogens, and, in particular, on the magnitude and the sign of dielectric anisotropy.

\section{Material}

The object of the study was a liquid crystal complex tris[1-(4-(4-propylcyclohexyl)phenyl)octane-1,3-dion][5,5'-di(heptadecyl)-2,2'-bipyridine] terbium $\left(\mathrm{Tb}\left(\mathrm{CPDk}_{3-5}\right)_{3} \mathrm{Bpy}_{17-17}\right)$, containing ligands similar to the previously studied lanthanidomesogens $\mathrm{Yb}\left(\mathrm{CPDk}_{3-5}\right)_{3} \mathrm{Bpy}_{17-17}[15,16]$. The synthetic schemes for the investigated materials are shown in Fig. 1.

Quantum-chemical simulation of the equilibrium geometry of $\mathrm{Yb}\left(\mathrm{CPDk}_{3-5}\right)_{3} \mathrm{Bpy}_{17-17}$ complexes was performed using the Prird 06 software by the DFT method with the PB exchange correlation functional (Fig. 2). 


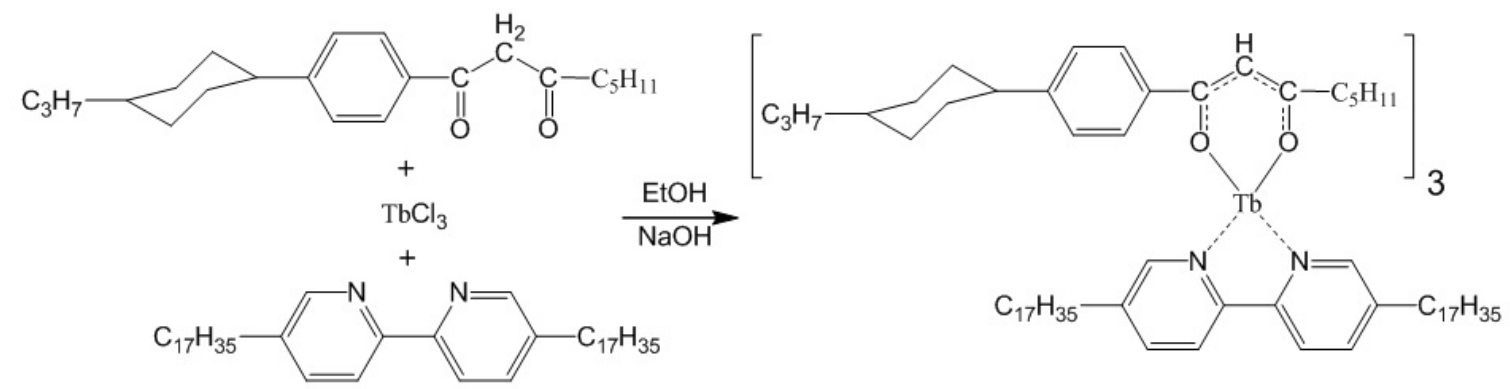

FIG. 1. Synthesis and chemical structure of the investigated terbium-based liquid crystalline complex

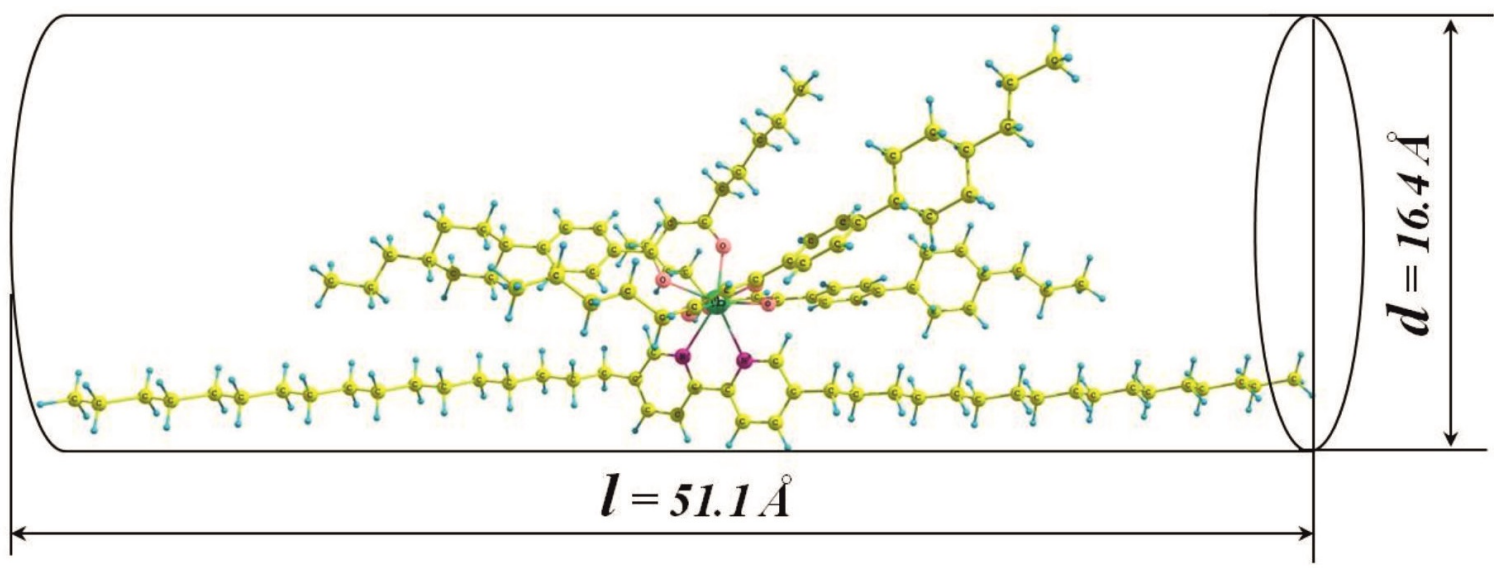

FIG. 2. Optimized structure and geometry parameters of $\mathrm{Tb}\left(\mathrm{CPDk}_{3-5}\right)_{3} \mathrm{Bpy}_{17-17}$ complexes

For synthesizing $\mathrm{Tb}\left(\mathrm{CPDk}_{3-5}\right)_{3} \mathrm{Bpy}_{17-17}$, an alcoholic solution of $0.031 \mathrm{~g}(0.083 \mathrm{mM}) \mathrm{TbCl}_{3} 6 \cdot \mathrm{H}_{2} \mathrm{O}$, while stirring, was slowly poured into a hot solution, containing $0.085 \mathrm{~g}(0.25 \mathrm{mM})$ of $\beta$-diketone (1-(4-(4-propylcyclohexyl)phenyl)octane-1,3-dion), $0.053 \mathrm{~g}(0.083 \mathrm{mM})$ 5,5'-diheptadecyl-2,2'-bipyridine and $0.015 \mathrm{~g}(0.25 \mathrm{mM}) \mathrm{EOH}$. The deposited yellow precipitate was filtered off while hot, under stirring, washed with alcohol and dried in vacuo. Yield: $0.078 \mathrm{~g}(52 \%)$. Formula: $\mathrm{C}_{113} \mathrm{H}_{175} \mathrm{~N}_{2} \mathrm{O}_{6} \mathrm{~Tb}$; Found, \%: $\mathrm{C}, 74.68 ; \mathrm{H}$, 9.84; N, 1.51; Tb, 8.72. Calculated, \%: C, 74.71; H, 9.71; N, 1.54; Tb, 8.75. IR spectrum, $\nu, \mathrm{cm}^{-1}: 1590,1492$ $\left(\mathrm{C}=\mathrm{N}\right.$, Phen); 1542, 1465, 1437, $938(\mathrm{C}=\mathrm{O}) ; 1394,1348,1024\left(\mathrm{CH}_{3}\right) ; 1172,784,765\left(\left(\mathrm{CH}_{2}\right) \mathrm{n}\right) ; 1254(\mathrm{C}=\mathrm{C})$, $1218,1103\left(\mathrm{C}_{6} \mathrm{H}_{4}\right) ; 729,654,634,602,512(\mathrm{~Tb}-\mathrm{O}) ; 728\left(\left(\mathrm{CH}_{2}\right)\right.$ n-O). CHN elemental microanalysis was performed with the analyzer CE Instruments EA-1110, X-ray fluorescence analysis - with the universal spectrometer "SUR-02 "Renom FV". IR Specta of the sample in KBr were registered with ALPHA FT-IR spectrometer. The terbium complex $\mathrm{Tb}\left(\mathrm{CPDk}_{3-5}\right)_{3} \mathrm{Bpy}_{17-17}$ forms a nematic liquid crystalline phase within the temperature range $100-160^{\circ} \mathrm{C}$.

The anisotropy of the magnetic susceptibility of the complex $\Delta \chi=\chi_{\|}-\chi_{\perp}\left(\chi_{\|}\right.$and $\chi$ are values of the magnetic susceptibility of the liquid crystal in the directions parallel and perpendicular to the axis of preferential orientation of molecules, respectively) was measured using the Faraday method [17]. The sign of $\Delta \chi$ was via optical birefringence measurements in a magnetic field (Cotton-Mouton effect) [13].

The obtained value $\Delta \chi$, that equals $-12690 \times 10^{-6} \mathrm{~cm}^{3} / \mathrm{mol}$, is one of the largest absolute values among the compounds using the same type of ligand environment but various lanthanide ions [13].

\section{Dielectric measurements}

The measurement of the dielectric permittivity is a powerful tool for characterizing the structure and physical properties of liquid crystalline materials [18] Investigations of the terbium complex were carried out using the analyzer HIOKI-3532 operating within the frequency range $100 \mathrm{~Hz}$ to $5 \mathrm{MHz}$. A titanium plane capacitor with the $200 \mu \mathrm{m}$ distance between electrodes (electrical capacity $12 \mathrm{pF}$ ) was used as a sample cell. The macroscopic alignment of the liquid crystal sample in the cell was provided by a magnetic field of up to 5000 Oe. As probing 
voltage $\mathrm{U}$ we used $1 \mathrm{~V}$ because the dielectric permittivity $\varepsilon$ do not depend on $\mathrm{U}$ between $0.5 \mathrm{~V}$ and $2.0 \mathrm{~V}$ (Fig. 3). In this range the electric field does not affect the orientation of the complex.

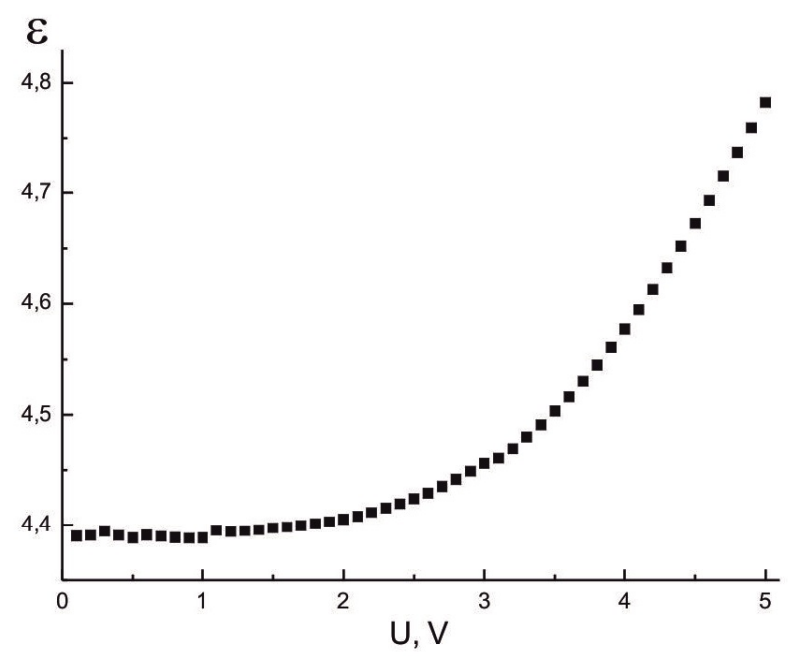

FIG. 3. The dielectric permittivity $\varepsilon$ of the sample versus the probing voltage $U$ (frequency $\left.f=10 \mathrm{kHz}, T=130{ }^{\circ} \mathrm{C}\right)$

X-ray diffraction pattern of a number of paramagnetic lanthanidomesogens oriented throughout a magnetic field showed that, unlike diamagnetic liquid crystal, the director (the preferred direction of orientation of the molecular long axes) can vary by several degrees from the direction of the highest value of the magnetic susceptibility $\chi$ [17]. The difference of 10 degrees between the directions of the axes of the highest value of the magnetic susceptibility $\chi$ and the highest value of the dielectric permittivity $\varepsilon$, has been found in the study of the dielectric properties of $\mathrm{Yb}\left(\mathrm{CPDk}_{3-5}\right)_{3} \mathrm{Bpy}_{17-17}$ using the orienting magnetic field [16]. Therefore, in this study we measured the dielectric permittivity $\mathrm{Tb}\left(\mathrm{CPDk}_{3-5}\right)_{3} \mathrm{Bpy}_{17-17}$ at different angles $\varphi$ between the directions of the probing electric field $\mathbf{E}(f=10 \mathrm{kHz})$ and the orienting magnetic field $(\mathbf{H}=5000 \mathrm{Oe})$. The dependence of $\varepsilon$ on $\varphi$ at $130^{\circ}$ is shown in Fig. 4.

It can be seen the dielectric permittivity reaches a maximum value at angle $\varphi=0^{\circ}$, i.e. the directions of the electric and magnetic fields, respectively, coincide $(\mathbf{E}|| \mathbf{H})$. The minimum value of the dielectric permittivity corresponds to the angle $\varphi=90^{\circ}$, if the direction of the electric field is orthogonal to the magnetic field $(\mathbf{E} \perp \mathbf{H})$. Consequently, the maximum of the magnetic susceptibility coincides with the maximum of the dielectric permittivity. That is why the measurement of the components of the dielectric permittivity $\mathrm{Tb}\left(\mathrm{CPDk}_{3-5}\right)_{3} \mathrm{Bpy}_{17-17}$ was carried out for $\mathbf{E}|| \mathbf{H}$ and $\mathbf{E} \perp \mathbf{H}$.

The orientation of the nematic liquid crystal molecules in the plane sample cell relative to the direction of the orienting magnetic field $\mathbf{H}$ and the probing electric field $\mathbf{E}$ is shown schematically in Fig. 5.

In the case of a positive sign of the magnetic susceptibility anisotropy $\Delta \chi>0$, the long axes of the mesogenic molecules are homogeneously aligned along the direction of the magnetic field (Fig. 6a,b). The paramagnetic complex under study, as noted above, has a negative sign of the macroscopic anisotropy of the magnetic susceptibility [13]. In this case the long axes of the molecules $\mathrm{Tb}\left(\mathrm{CPDk}_{3-5}\right)_{3} \mathrm{Bpy}_{17-17}$ are perpendicular to the orienting magnetic field (Fig. 5c,d). However, the magnetic field does not provide a homogeneous orientation (same direction) rather a planar one of the long molecular axes throughout the sample (Fig. 5c,d). Fig. 5 shows that for $\mathbf{E}|| \mathbf{H}$ the electric field direction is perpendicular to the long axes of the molecules. Then it seems evident that the measured component $\varepsilon_{(\mathbf{E} \mid \mathbf{H})}$ is the transverse component of the dielectric permittivity $\varepsilon_{\perp}$. The reorientation around the short molecular axes is at lower frequencies, probably hidden under the conductivity part.

The measurement of the dielectric permittivities over frequency range of $100 \mathrm{~Hz}$ to $5 \mathrm{MHz}$ indicated the presence of the dispersion within the temperature range of the nematic phase and in the isotropic liquid state $\varepsilon_{i s}$. To illustrate it, Fig. 6 showed the dependencies of $\varepsilon_{(\mathbf{E}|| \mathbf{H})}=\varepsilon_{\perp}$ and $\varepsilon_{(E \perp H)}$ on $\lg f$ at $130{ }^{\circ} \mathrm{C}$, and also $\varepsilon_{i s}$ at $160^{\circ} \mathrm{C}$.

First, it can be seen from Fig. 6 that within the $100 \mathrm{~Hz}-100 \mathrm{kHz}$ frequency range the anisotropy $\varepsilon_{(\mathbf{E} \perp \mathbf{H})}-\varepsilon_{\perp}$ has a negative sign and changes the sign to positive at the highest frequencies (the insert in Fig. 6). The increase of all components $\varepsilon_{\perp}, \varepsilon_{(\mathbf{E} \perp \mathbf{H})}$ and $\varepsilon_{i s}$ at frequencies below $5 \mathrm{kHz}$ is due to contribution of conductivity to the 


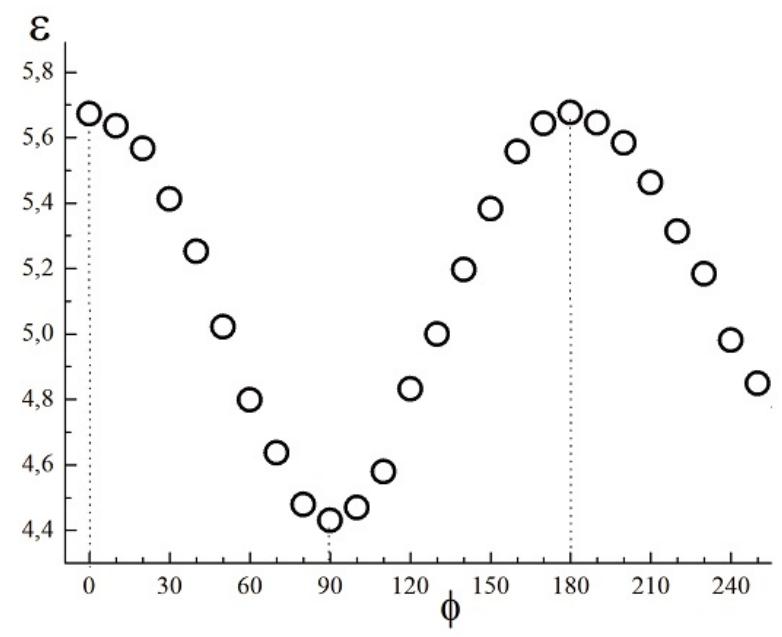

FIG. 4. The dielectric permittivity $\varepsilon$ of the sample versus the angle $\varphi$ between the directions of the probing electric field $\mathbf{E}(f=10 \mathrm{kHz})$ and the direction of orienting magnetic field $(\mathbf{H}=5000$ Oe $), T=130{ }^{\circ} \mathrm{C}$
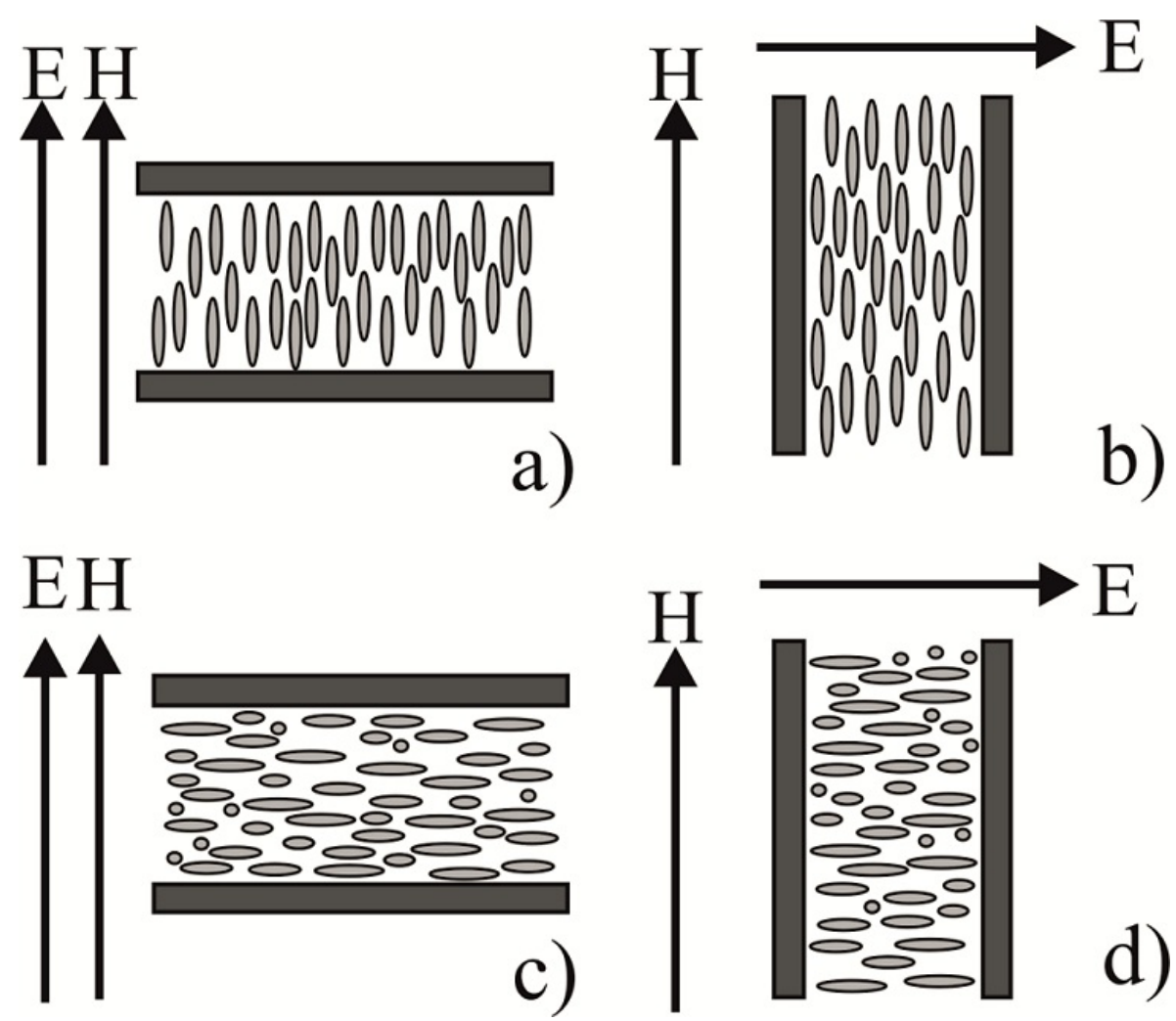

FIG. 5. The orientation of nematic liquid crystal molecules in the sample cell under the influence of the magnetic field $\mathbf{H}$ relative to the direction of the probing electric field $\mathbf{E}$ : a) and b) nematic liquid crystal with a positive magnetic anisotropy $\mathbf{E}|| \mathbf{H}$ and $\mathbf{E} \perp \mathbf{H}$ respectively; c) and d) - nematic liquid crystal with a negative magnetic anisotropy $\mathbf{E}|| \mathbf{H}$ and $\mathbf{E} \perp \mathbf{H}$ respectively 


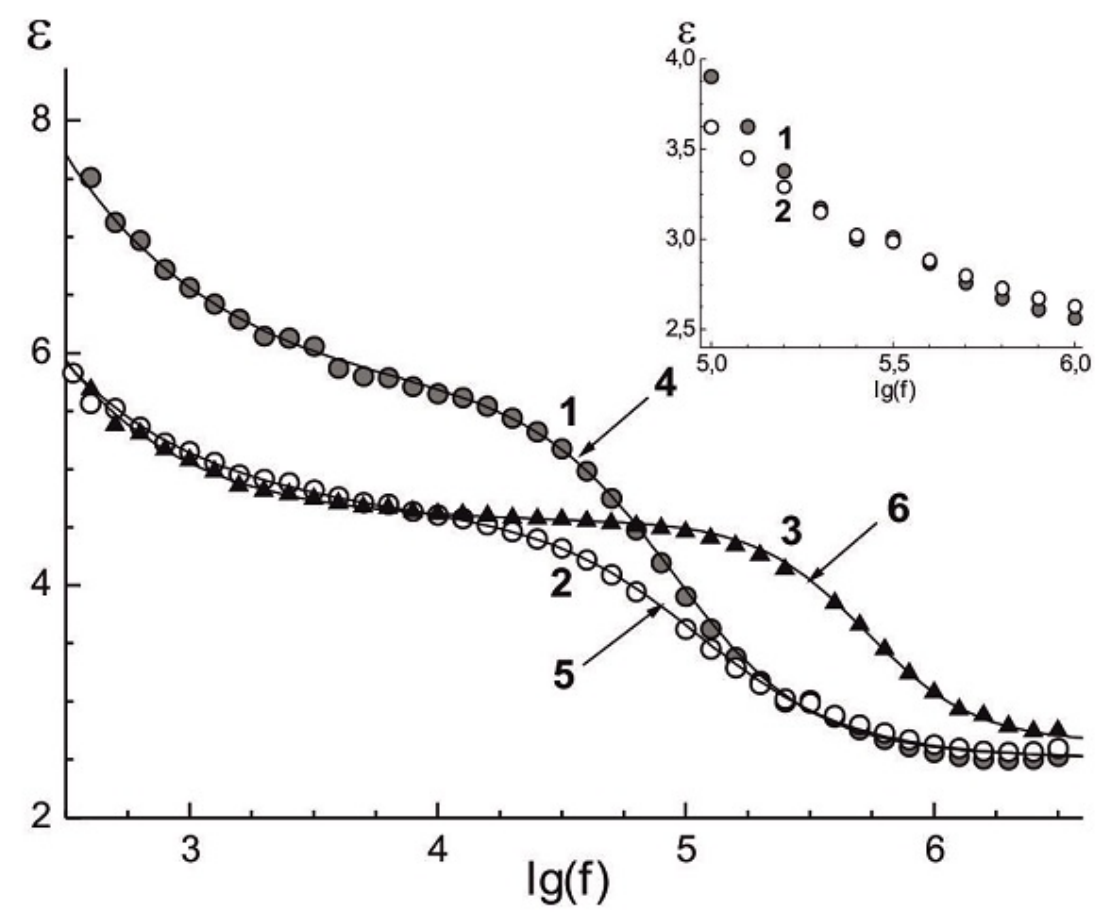

FIG. 6. The dependence of the components of dielectric permittivity on the electric field frequency. Experimental points: $1-\varepsilon_{(\mathbf{E} \mid \mathbf{H})}=\varepsilon_{\perp} ; 2-\varepsilon_{(\mathbf{E} \perp \mathbf{H})} ; 3-\varepsilon_{i s}$; Theoretical curves calculated using the Cole-Cole equation: $4-\varepsilon_{(\mathbf{E}|| \mathbf{H})}=\varepsilon_{\perp} ; 5-\varepsilon_{(\mathbf{E} \perp \mathbf{H})} ; 6-\varepsilon_{i s}$

effective value of the dielectric permittivity. From the given data it also follows that the dispersion is realized within the same frequency range $10 \mathrm{kHz}-1 \mathrm{MHz}$. The values $\varepsilon_{\perp}=2.496$ and $\varepsilon_{(\mathbf{E} \perp \mathbf{H})}=2.564$ at highest frequency $f=5 \mathrm{MHz}$ differ slightly from the values $\mathrm{n}_{o}^{2}=2.256$ and $\mathrm{n}_{e}^{2}=2.522$, that was measured using the prism method [19] at wavelength $\lambda=632 \mathrm{~nm}$ ). This means that the dipole part is almost completely excluded from the dielectric polarization of the complex at frequencies above $5 \mathrm{MHz}$. Perhaps the small contribution $\left(\varepsilon_{\perp}-\mathrm{n}_{o}^{2}\right)$ and $\left(\varepsilon_{(\mathbf{E} \perp \mathbf{H})}-\mathrm{n}_{e}^{2}\right)$ to the dielectric polarization by intramolecular rotation of polar groups remains. In order to understand the observed phenomenon, it is useful to consider the well-known dipole mechanisms of dielectric polarization of nematic liquid crystals [20-22]. In the presence of the longitudinal and transverse components of the molecular dipole moment these mechanisms are: the rotation of molecules around short molecular axes, the rotation around the long axes and the rotational motion about the long axis of the precessing molecule. It should be noted that the rotation of molecules around the short transverse axis requires overcoming a potential barrier responsible for a long-range order in the liquid crystal phase, while the rotation of molecules around the longitudinal molecular axes and the cone is less hindered. Therefore, parts of the dipole polarization $\varepsilon_{\perp}$ and $\varepsilon_{\|}$are excluded from the polarization at much higher frequencies than the dipole part of polarization $\varepsilon_{\|}$determined by the first mechanism. Therefore, it can be claimed that the experimentally observed coincidence of dispersion areas of the permittivity $\varepsilon_{\perp}$ and $\varepsilon_{(\mathbf{E} \perp \mathbf{H})}$ of $\mathrm{Tb}\left(\mathrm{CPDk}_{3-5}\right)_{3} \mathrm{Bpy}_{17-17}$ (Fig. 6) indicates that the dipole parts of the dielectric polarization of the complex $\varepsilon_{\perp}$ is associated with the rotation of the polar molecules around the longitudinal axes and across to the cone surface. Reorientation around the short molecular axes was not seen. Perhaps this mechanism of the complex dielectric polarization is realized at frequencies below $100 \mathrm{~Hz}$ and may be hidden under the conductivity part.

The experimental frequency dependences of the dielectric permittivities given in Fig. 6 are approximated by the Cole-Cole equation taking into account the contribution of the sample's conductivity:

$$
\varepsilon=\varepsilon_{\infty}+\frac{\varepsilon_{0}-\varepsilon_{\infty}}{1+(i 2 \pi f \tau)^{1-\alpha}}+\frac{B}{f^{N}},
$$

where, $\varepsilon_{0}$ is a quasi-static dielectric permittivity; $\varepsilon_{\infty}$ is a high-frequency value of the dielectric permittivity; $\tau$ is average time of the dielectric relaxation; $\alpha$ is a parameter characterizing the distribution of relaxation times; $\mathrm{B}$ and $\mathrm{N} \leq 1$ are numerical coefficients. The best agreement between theoretical curves and experimental points for the components of the dielectric permittivity was obtained under the following parameters: for $\varepsilon_{\perp}\left(\varepsilon_{0}=5.6 ; \varepsilon_{\infty}=2.5\right.$; 
$\left.\alpha=0.1 ; \tau=1.7 \times 10^{-6} ; B=100 ; N=0.67\right)$, for $\varepsilon_{(\mathbf{H} \perp \mathbf{E})}-\left(\varepsilon_{0}=4.6 ; \varepsilon_{\infty}=2.5 ; \alpha=0.15 ; \tau=1.4 \times 10^{-6}\right.$; $B=100 ; N=0.75)$, and for $\varepsilon_{i s}\left(\varepsilon_{0}=4.55 ; \varepsilon_{\infty}=2.65 ; \alpha=0.01 ; \tau=1.4 \times 10^{-6} ; B=100 ; N=0.75\right)$. In Fig. 6 theoretical curves are represented by solid lines 4,5,6. The above parameters are also determined for other temperatures in the nematic and isotropic phases.

The dependencies of quasi-static values $\varepsilon_{0 \perp}=\varepsilon_{0(\mathbf{E}|| \mathbf{H})}$, and $\varepsilon_{0 i s}$ on the temperature are shown in Fig. 7, where the lower scale presents temperature; and the upper scale presents the relative temperature $\Delta T$.

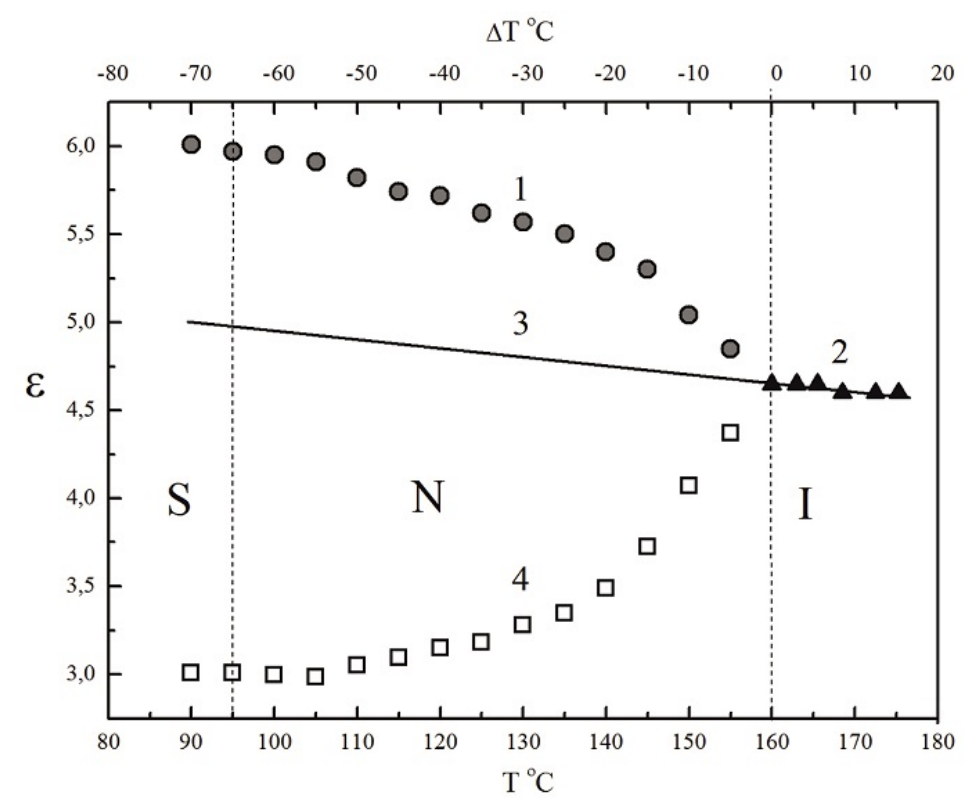

FIG. 7. The dependence of the quasi-static dielectric permittivity components on the temperature: $1-\varepsilon_{o \perp} ; 2-\varepsilon_{o i s} ; 3-<\varepsilon>; 4-\varepsilon_{o \|}$

The value $\varepsilon_{\|}$can be calculated using the Maier-Meier theory of liquid crystal dielectric polarization [23] and experimental data showing that the average value of the dielectric permittivity $<\varepsilon>=\left(\varepsilon_{\|}+2 \varepsilon_{\perp}\right) / 3$ equals $\varepsilon_{i s}$ or is only a few percent different from it at the transition temperature from an isotropic phase to a mesomorphic state. The extrapolation of the temperature dependence $\varepsilon_{o i s}$ to the area of the mesophase existence allows one to obtain the dependency of $\langle\varepsilon\rangle$ on the temperature (Fig. 7) and calculating $\varepsilon_{\|}$at different temperatures. Thus determined values of $\varepsilon_{o \|}$ are presented in Fig. 7 (dependence 4). The value of the dielectric anisotropy of the complex $\mathrm{Tb}\left(\mathrm{CPDk}_{3-5}\right)_{3} \mathrm{Bpy}_{17-17} \Delta \varepsilon=\varepsilon_{\|}-\varepsilon_{\perp}$, calculated on the basis of the data shown in Fig. 7 varies from -0.5 to -2.15 within the temperature $\Delta T$ from $-5{ }^{\circ} \mathrm{C}$ to $-25{ }^{\circ} \mathrm{C}$ For the studies in the paper [6], the ytterbiumbased coordination compound $\mathrm{Yb}\left(\mathrm{CPDk}_{3-5}\right)_{3} \mathrm{Bpy}_{17-17}$ with similar ligands in the same interval of temperatures $\Delta T$ the dielectric anisotropy $\Delta \varepsilon$ varies from -0.4 to -2.4 . We can conclude that the dielectric anisotropies of the discussed liquid crystal complexes, found within the same frequency range, are identical in sign and close in magnitude.

During the analysis of the dispersion of the dielectric permittivities of the studied complex $\varepsilon_{\perp}$ and $\varepsilon_{(\mathbf{E} \perp \mathbf{H})}$ the relaxation times for different temperatures were determined. The dependencies of relaxation times $\tau_{\perp} \tau_{(\mathbf{E} \perp \mathbf{H})}$ and $\tau_{\text {is }}$ on the reciprocal temperature $1 / T$ are shown in Fig. 8.

From the slopes of the lines according to the Arrhenius equation:

$$
\tau=\tau_{0} \exp \left(\frac{W}{k T}\right)
$$

we obtained the activation energy of the molecular mechanisms responsible for the dipole polarization in the studied frequency range in the mesophase and isotropic state. The values $\mathrm{W}_{\|}$and $\mathrm{W}_{(\mathbf{E} \perp \mathbf{H})}$ are identical within experimental error, and equal to $80 \mathrm{~kJ} / \mathrm{mol}$. The activation energy in the isotropic phase was greater $\mathrm{U}_{i s}=105 \mathrm{~kJ} / \mathrm{mol}$. This can be explained by the fact that the rotation of the molecules around the long axis in the isotropic phase is more hindered than in the nematic phase. As an example this was confirmed by experimental data for 4,4'-di-nheptyloxyazoxybenzene [24].

The significant difference between the values of quasi-static dielectric permittivity $\varepsilon_{\|} \varepsilon_{\perp} \varepsilon_{i s}$ and refractive indices $n_{e}, n_{o}$ and $n_{i s}$, and also the presence of dispersion $\varepsilon_{(\mathbf{E} \perp \mathbf{H})}, \varepsilon_{\perp}, \varepsilon_{i s}$ suggests that the studied paramagnetic 


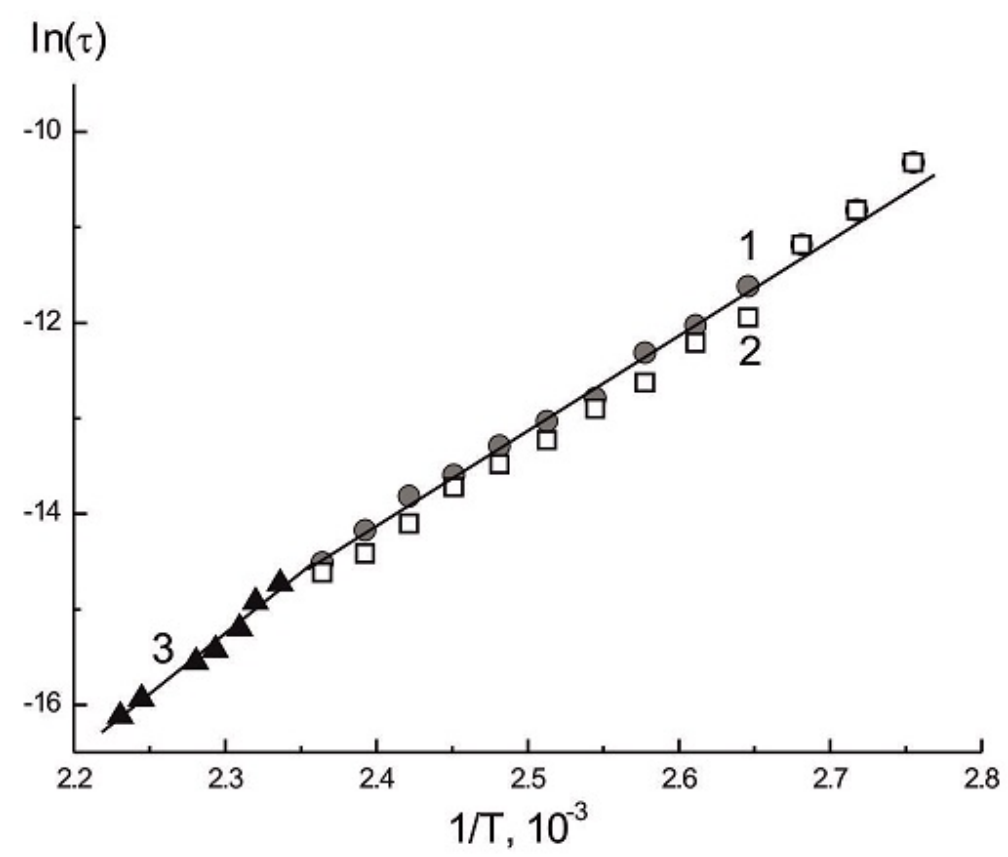

FIG. 8. The dependence of relaxation times on the temperature. $1-\tau_{\perp} ; 2-\tau_{(\mathbf{E} \perp \mathbf{H})} ; 3-\tau_{i s}$

complex is a polar substance. The value of the permanent dipole moment of the complex $\mu=6.4 \mathrm{D}$ was determined using the dielectric permittivity $\varepsilon_{i s}=4.7$, the refractive index of the isotropic phase $n=1.522$ and the Onsager's formula:

$$
\frac{9\left(\varepsilon_{i s}-n^{2}\right)\left(2 \varepsilon_{i s}+n^{2}\right)}{\varepsilon_{i s}\left(n^{2}+2\right)^{2}}=\frac{4 \pi N_{A} \rho \mu^{2}}{M k T},
$$

where $N_{A}$ - Avogadro's number, $\rho \approx 1 \mathrm{~g} / \mathrm{cm}^{3}$ - complex density, $M=1814$ - molecular weight. The true value of the dipole moment of the complex $\mu$ can be higher than the obtained one, as the Onsager's formula is valid only for weakly polar substances. In addition, the presence of the longitudinal component of the dipole moment, that has not manifested itself during the polarization within the $100 \mathrm{~Hz}-5 \mathrm{MHz}$ electric field frequency range, can also increase $\mu$.

\section{Conclusion}

Dispersions of the dielectric permittivity components of $\mathrm{Tb}\left(\mathrm{CPDk}_{3-5}\right)_{3} \mathrm{Bpy}_{17-17}$ and $\mathrm{Yb}\left(\mathrm{CPDk}_{3-5}\right)_{3} \mathrm{Bpy}_{17-17}$ are realized within the same frequency range and are associated with the rotation of the polar molecules around the longitudinal axes and across to the cone's surface. Dispersion associated with reorientation around the short molecular axes was not seen. The value dielectric anisotropies of the discussed liquid crystal complexes are identical in sign and close in magnitude, therefore it can be concluded that variation of the central ion has a weak influence dielectric properties of lanthanidomesogens with the same ligand environment.

\section{Acknowledgements}

L. A. Dobrun, A. P. Kovshik, A. S. Sakhatskii thank grant SPbU No 11.37.161.2014 for support of the investigation of the dielectric properties of the terbium-based complex.

E. I. Ryumtsev thanks grant SPbU No 11.37.145.2014 for support of the investigation of the optical properties of the terbium-based.

A. S. Krupin, A. A. Knyazev, Yu. G. Galyametdinov thank grant RSF14-13-00758 for support of the synthesis of the terbium-based. 


\section{References}

[1] Feng J., Zhang H. Hybrid materials based on lanthanide organic complexes: a review. Chemical Society Reviews, 2013, 42, P. 387-410.

[2] Kalyani N.T, Dhoble S.J. Novel materials for fabrication and encapsulation of OLEDs. Renewable and Sustainable Energy Reviews, 2015, 44, P. 319-347.

[3] Xu H., Sun Q., An Z., Wei Y., Liu X. Electroluminescence from europium (III) complexes. Coordination Chemistry Reviews, 2015, 293, P. 228-249.

[4] Knyazev A.A., Molostova E.Yu., Krupin A.S., Heinrich B., Donnio B., Haase W., Galyametdinov Yu.G. Mesomorphic behaviour and luminescent properties of mesogenic-diketonate lanthanide adducts with 5,5/-di(heptadecyl)-2,2/-bipyridine. Liquid Crystals, 2013, 40, P. 857-863.

[5] Binnemans K. Lanthanidomesogens. Handbook on the Physics and Chemistry of Rare Earths, 2013, 43, P. 1-158

[6] Binnemans K. Luminescence of metallomesogens in the liquid crystal state Journal of Materials Chemistry, 2009 , 19, P. 448-453.

[7] Goossens K., Bruce D.W., Van Deun R., Binnemans K., Cardinaels Th. Nematogenic tetracatenar lanthanidomesogens. Dalton Transactions, 2012, 41, P. 13271-13273.

[8] Terazzi E., Suarez S., Torelli S., Nozary H., Imbert D., Mamula O., Rivera J.-P., Guillet E., Bénech J.-M., Bernardinelli G., Scopelliti R., Donnio B., Guillon D., Bünzli J.-C., Piguet C. Introducing Bulky Functional Lanthanide Cores into Thermotropic Metallomesogens: A Bottom-Up Approach. Advanced Functional Materials, 2006, 16, P. 157-168.

[9] Binnemans K., Lodewyckx K., Parac-Vogt T.N., R.Van Deun, Goderis B., Tinant B., K. Van Hecke, L. Van Meervelt. Adducts of Schiff Bases with Tris ( $\beta$-diketonato) lanthanide(III) Complexes: Structure and Liquid-Crystalline Behaviour. European Journal of Inorganic Chemistry, 2003, P. 3028-3033.

[10] Knyazev A.A., Krupin A.S., Molostova E.Yu, Romanova K.A., Galyametdinov Yu.G. Influence of Structural Anisotropy on Mesogenity of Eu (III) Adducts and Optical Properties of Vitrified Films Formed on their Base. Inorganic Chemistry, 2015, 54, P. 8987-8993.

[11] Galyametdinov Yu.G., Knyazev A.A., Dzhabarov V.I., Cardinaels T., Driesen K.S., Görller-Walrand C., Binnemans K. Polarized luminescence from aligned samples of nematogenic lanthanide complexes. Advanced materials, 2008, 20, P. $252-257$.

[12] Dzhabarov V.I., Knyazev A.A., Strelkov M.V., Molostova E.Yu., Schustov V.A., Haase W., Galyametdinov Yu.G. Tris ( $\beta$-diketonates) lanthanum nematic adducts. Liquid Crystals, 2010, 37, P. 285-291.

[13] Dzhabarov V.I., Knyazev A.A., Nikolaev V.F., Galyametdinov Yu.G. Anisotropy of the magnetic susceptibility of mesogeneous lanthanide complexes. Russian Journal of Physical Chemistry A, 2011, 85, P. 1450-1453.

[14] Mironov V.S., Galyanetdinov Yu.G., Ceulemans A., Gorller-Walrand C., Binnemans K. Influence of crystal-field perturbations on the room-temperature magnetic anisotropy of lanthanide complexes, Chem. Phys. Lett., 2001, 345, P. 132-140.

[15] Dobrun L.A., Sakhatskii A.S., Kovshik A.P., Ryumtsev E.I., Knyazev A.A., Galyametdinov Yu.G. Dielectric Properties of an YtterbiumBased Nematic Liquid-Crystal Complex. JETP Letters, 2014, 99, P. 133-135.

[16] Dobrun L.A., Sakhatskii A.S., Kovshik A.P., Ryumtsev E.I., Kolomiets I.P., Knyazev A.A., Galyametdinov Yu.G. Dielectric and Magnetic Anisotropy of a Nematic Ytterbium Complex. JETP, 2015, 147, P. 1064-1067.

[17] Galyametdinov Y.G., Haase W., Goderis B., Moors D., Driesen K., R. van Deun, Binnemans K. Magnetic alignment study of rare-earthcontaining liquid crystals. Journal of Physical Chemistry B, 2007, 111, P. 13881-13885.

[18] Haase W., Wróbel S. Relaxation phenomena: liquid crystals, magnetic systems, polymers, high-Tc superconductors, metallic glasses. Springer Science \& Business Media, 2013.

[19] Kovshik A.P., Krainyukov E.S., Kovshik S.A., Knyazev A.A., Galyametdinov Yu.G., Ryumtsev E.I. Optical Anisotropy of Liquid Crystal Lanthanide Complexes. Optics and Spectroscopy, 2014, 116, P. 56-61.

[20] Nordio P.L., Rigatti G., Serge U. Dielectric relaxation theory in nematic liquids. Molecular physics, 1973,25, P. $129-136$.

[21] Tsvetkov V.N., Ryumtsev E.I., Polushin S.G., Kovshik A.P. Molecular mechanisms of polarization and its relaxation in nematic liquidcrystals. Acta physica polonica A, 1979, 56, P. 871-878.

[22] Clark M.G. Macroscopic Properties of Liquid Crystals. Molecular Crystals and Liquid Crystals, 1985,127, P. 141.

[23] Maier W., Meier G. Eine einfache Theorie der dielektrischen Eigenschaften homogen orientierter kristallinflüssiger Phasen des nematischen Typs. Z. Naturforschg, 1961, 16a, P. 262267.

[24] Nguyen X.P., Urban S., Wrobel S., Kresse H. Acta physica polonica, 1978, A54(5), P. 617-623. 